\title{
The role of MHC genes in contagious cancer: the story of Tasmanian devils
}

\author{
Alison Caldwell ${ }^{1} \cdot$ Hannah V. Siddle ${ }^{1}$
}

Received: 19 April 2017 /Accepted: 21 April 2017 /Published online: 11 July 2017

(C) The Author(s) 2017. This article is an open access publication

\begin{abstract}
The Tasmanian devil, a marsupial species endemic to the island of Tasmania, harbours two contagious cancers, Devil Facial Tumour 1 (DFT1) and Devil Facial Tumour 2 (DFT2). These cancers pass between individuals in the population via the direct transfer of tumour cells, resulting in the growth of large tumours around the face and neck of affected animals. While these cancers are rare, a contagious cancer also exists in dogs and five contagious cancers circulate in bivalves. The ability of tumour cells to emerge and transmit in mammals is surprising as these cells are an allograft and should be rejected due to incompatibility between Major Histocompatibility Complex (MHC) genes. As such, considerable research has focused on understanding how DFT1 cells evade the host immune system with particular reference to MHC molecules. This review evaluates the role that MHC class I expression and genotype plays in allowing DFT1 to circumvent histocompatibility barriers in Tasmanian devils. We also examine recent research that suggests that Tasmanian devils can mount an immune response to DFT1 and may form the basis of a protective vaccine against the tumour.
\end{abstract}

Keywords Contagious cancer · DevilFacial TumourDisease · MHC downregulation $\cdot$ MHC $\cdot$ Vaccine

This article is published in the Special Issue MHC Genes and Their Ligands in Health and Disease with Editor Prof. Ronald Bontrop.

Hannah V. Siddle

H.V.Siddle@soton.ac.uk

1 Department of Biological Science, University of Southampton, Highfield Campus, Southampton SO17 1BJ, UK

\section{Introduction}

Cells transferred between individuals should be rejected in a robust immune response due primarily to differences in Major Histocompatibility Complex (MHC) genes between individuals (Gorer 1938; Gorer et al. 1948; Snell and Kelton 1953; Dausset et al. 1965; Benacerraf 1992). Tumour cells are no exception, and seminal experiments by Gorer demonstrated that tumour allografts are rejected by host animals (Gorer 1938; Gorer et al. 1948). However, in some species, tumours have emerged that can propagate between individuals, becoming true contagious cancers. The transmission of cancer cells as an allograft in vertebrates is surprising and contradicts our understanding of how histocompatibility barriers function to prevent the transfer of cells between individuals.

In the context of a contagious cancer, cells passing as an allograft should be recognised by T-cells stimulated by allogeneic MHC class I and/or class II molecules present on the cancer cells (Gould and Auchincloss 1999). The stimulation of T-cells by allogeneic MHC molecules is termed direct recognition and initiates a rapid immune response that occurs within 7 to 14 days and results in T-cell infiltration to the graft (Waanders et al. 2007). The transfer of contagious cancer cells should also initiate indirect recognition of the tumour cells where T-cells are stimulated by the presentation of foreign peptides on self antigen presenting cells (APCs) (Dausset 1981; Snell 1981). This process should occur in contagious cancers as cells divide and die in growing tumours, shedding foreign proteins that are taken up by APCs and presented in the context of MHC class II. These foreign peptides could derive from the donor $\mathrm{MHC}$ as this is a common event during graft rejection (Benichou et al. 1992). The frequency of T-cells involved in indirect recognition is 100-fold lower than direct recognition but plays a role in chronic graft rejection (Liu et al. 1993). APCs that are transferred to the host 
along with tumour cells may also play a role where allogeneic MHC molecules are recognised. Transfer of APCs would also introduce MHC molecules from a third individual, as these cells would derive from the most recent host, not the cancer. While this review will focus primarily on MHC-restricted recognition, other mechanisms are also relevant, including host antibodies that could recognise non-MHC antigens on the cancer cells.

Eight naturally occurring transmissible cancers have been found in wild species. In dogs (Canis lupus), Tasmanian devils (Sarcophilus harrisii) and four species of bivalves (Cerastoderma edule, Polititapes aureus, Mytilus trossulus and Mya arenaria), cancer cells are able to pass as an allograft between individuals (Fig. 1). Here we review the transmission of contagious cancers in the Tasmanian devil and the role of MHC class I molecules in this process.

\section{Examples of contagious cancers}

In total, four genetically distinct transmissible cancers have been found in bivalves known collectively as bivalve transmissible neoplasms (BTNs) (Metzger et al. 2015, 2016). The discovery of transmissible cancers in multiple bivalve species in the marine environment, including soft shell clams (M. arenaria), mussels (M. trossulus), cockles (C. edule) and golden carpet shell clams ( $P$. aureus), suggests that the transmission of cancer cells may be relatively common among bivalves (Metzger et al. 2015, 2016). These species are filter feeders and transmission experiments have shown that the cancer cells are passed through seawater. Most remarkably, species barriers do not necessarily confine these cancers and the BTN circulating in P. aureus derived from a distinct species, Venerupis corrugata.

Canine Transmissible Venereal Tumour (CTVT) is a transmissible cancer that arose in an ancient species of dog or wolf more than 10,000 years ago (Novinski 1876; Murchison et al. 2014). CTVT is spread between dogs during coitus and sniffing and licking (Karlson and Mann 1952), with tumours characterised by small, firm, localised nodules around the base of the glans penis of male dogs and in the vaginal vestibulum of female dogs (Murchison 2008). During its long evolution, CTVT has diverged into different genetic subtypes (Murchison et al. 2014) and has spread to all continents, with a higher prevalence in areas where neutering dogs is less common (Strakova and Murchison 2014). With the exception of infection in puppies or in immunocompromised canines, CTVT does not normally metastasize and is rarely fatal (Cohen 1973). The relatively benign nature of CTVT has meant that the tumour has become a form of parasite that coexists with its host species, the dog.

The Tasmanian devil is a carnivorous marsupial that is endemic to the island of Tasmania south of mainland Australia and is the only mammal in which two genetically distinct contagious cancers have emerged (Pearse and Swift 2006; Pye et al. 2016b). Devil Facial Tumour Disease 1 (DFT1) was first identified in the Tasmanian devil in 1996 (Pearse and Swift 2006), and in 2014, a second transmissible cancer Devil Facial Tumour 2 (DFT2) was discovered (Pye et al. 2016b). Both DFTs cause tumours primarily on the face, neck and oral cavity of the Tasmanian devil, and these tumours are spread through biting during feeding and mating

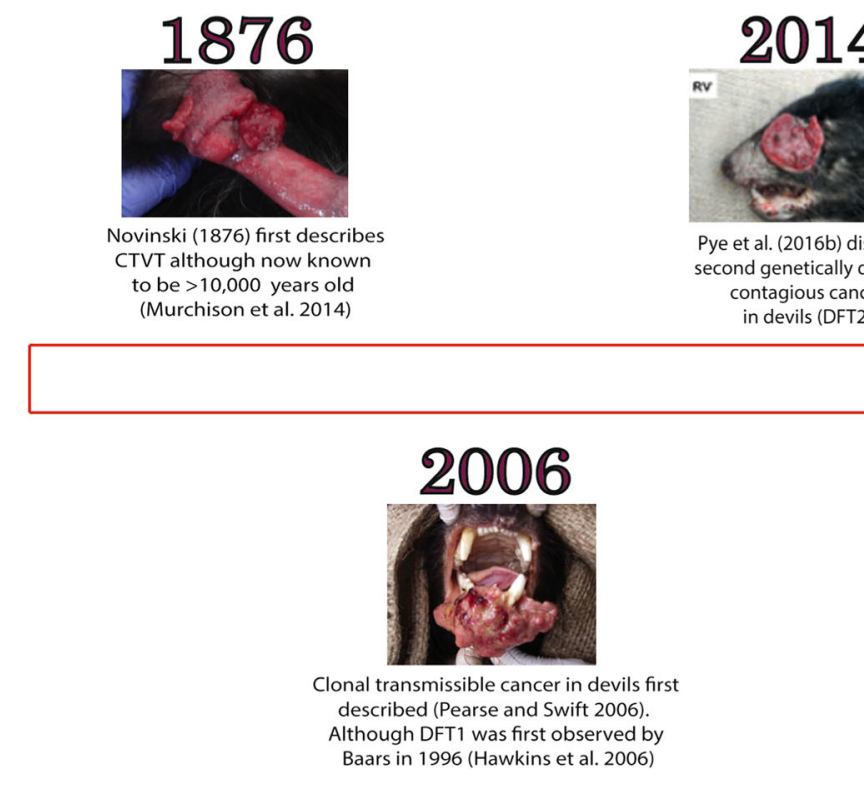

Fig. 1 Timeline of the discovery of the contagious cancers CTVT, DFT1, DFT2 and BTNs. The images presented were originally published as follows: CTVT—Siddle et al. (2015); DFT1—Siddle et al. (2015);

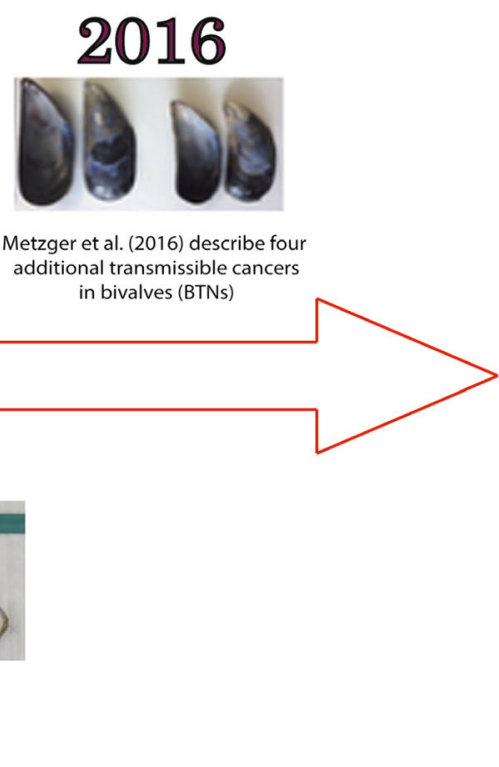

DFT2 - Pye et al. (2016b); clam leukaemia_-Metzger et al. (2015); BTNs-Metzger et al. (2016) 
behaviour (Hamede et al. 2008; Murchison 2008). The tumours are first visible as small nodules, usually on a mucosal surface, and after 6 months grow to become multilobed, infected and ulcerated (Loh et al. 2006). DFT1 has a high mortality rate with the first evidence of an immune response to the tumours reported only recently and in only six devils (Pye et al. 2016a). The mortality rate and severity of DFT2 are potentially similar to DFT1, but due to the small number of animals with the tumour, the mortality rate is difficult to determine with confidence (Pye et al. 2016b).

\section{History and emergence of DFT1 and DFT2}

In the 1990s, the Tasmanian devil was relatively common across Tasmania with an estimated population size of 130,000-150,000 devils (McCallum et al. 2007). However, in 1996, a nature photographer, Christo Baars, photographed a Tasmanian devil with a large facial tumour in north-eastern Tasmania (Hawkins et al. 2006). From 1996 to 2001, large facial tumours were observed on devils across the east of Tasmania and the disease was termed Devil Facial Tumour Disease (DFTD now DFT1). At present, DFT1 is present across almost the entire devil range, with only animals in north-west Tasmania disease free (Save the Tasmanian Devil Program 2016). In 2014, a second, genetically distinct contagious tumour, DFT2, was discovered and is thought to be restricted to the south-east of Tasmania (Pye et al. 2016b) (Fig. 1).

DFT1 and DFT2 have a similar gross morphology, but are genetically distinct tumours that most likely originated in different host Tasmanian devils (Pye et al. 2016b). The clonal origin of DFT1 was first proposed due to the highly similar karyotype rearrangements present in tumour samples from different animals (Pearse and Swift 2006). Genetic analysis of DFT1 confirmed that it is a monophyletic clonally transmissible tumour (Siddle et al. 2007; Murchison et al. 2010), and genome sequencing of two geographically distinct DFT1 tumours revealed that it is a relatively stable cell lineage (Murchison et al. 2012). Despite this stability, analysis of 104 DFT1 tumours shows that DFT1 has evolved by linear radiation of subtypes of DFT1 across Tasmania (Murchison et al. 2012).

The evidence that DFT2 is a distinct contagious cancer clone derives from cytogenetics and analysis of microsatellite markers. DFT1 has four unique marker chromosomes not found in host devil cells, and these marker chromosomes are also not found in four DFT2 tumours (Pye et al. 2016b). Further, DFT2 tumours carry a number of other cytogenetic abnormalities in comparison to DFT1 but all have an identical karyotype (Pye et al. 2016b). Interestingly, DFT2 carries a Y chromosome (Pye et al. 2016b) whereas DFT1 is of female origin with no traces of a $\mathrm{Y}$ chromosome (Murchison et al.
2012). Furthermore, DFT1 and DFT2 tumours have different genotypes at nine microsatellite markers and the tumours have different MHC genotypes.

\section{The MHC locus in the Tasmanian devil}

There is not yet a comprehensive map of the MHC region in the Tasmanian devil. However, four genomic regions of MHC class I and II genes have been assembled and annotated from Bacterial Artificial Chromosomes (BACs) contigs (Cheng et al. 2012b). All four regions map to chromosome $4 \mathrm{q}$ with two regions containing MHC class I genes and two regions containing MHC class II genes (Cheng et al. 2012b). This analysis implies that the organisation of MHC genes in the Tasmanian devil is similar to other marsupials, rather than eutherian mammals, with the MHC class I genes interspersed with genes involved with antigen processing, such as Transporters for Antigen Processing (TAP1 and TAP2) (Cheng et al. 2012b). Three classical MHC class I genes, Saha-UA, Saha-UB and Saha-UC, and two non-classical MHC class I genes, Saha-UD and Saha-UK, have been identified on the BACs (Cheng et al. 2012b), and three further non-classical MHC class I genes, Saha-UM, Saha-MRI and Saha-CD1, have been characterised (Cheng and Belov 2014). More recently, a sixth family of non-classical MHC class I genes, the Saha-UT family, was identified as a novel family of MHC class I genes unique to non-eutherian mammals (Papenfuss et al. 2015).

Saha-UA, Saha-UB and Saha-UC are classified as classical MHC class I genes due to ubiquitous expression and polymorphism in the peptide binding region, but these genes are not orthologous to HLA-A, HLA-B or HLA-C (Cheng et al. 2012b). Saha-UA, Saha-UB and Saha-UC derive from gene duplications in the devil lineage (Cheng et al. 2012b) and are very closely related genes (Lane et al. 2012). PCR amplification of the MHC class I peptide-binding region of Saha-UA, Saha-UB and Saha-UC identified six Saha-UA alleles, seven Saha-UC alleles and ten Saha-UB alleles (Lane et al. 2012). The MHC class I alleles in the devil are difficult to assign to loci due to their high sequence similarity; this is illustrated by the phylogenetic analysis in Fig. 2.

The class I genes, Saha-UD, Saha-UK, Saha-UM, SahaUT, Saha-MRI and Saha-CD1, have been classified as nonclassical due to tissue-specific expression, low levels of polymorphism and, in the case of Saha-MR1 and Saha-CD1, orthology to their human counterparts (Cheng and Belov 2014; Papenfuss et al. 2015). Saha-UD is expressed in blood, spleen and DFT1 cells and alleles have $97.7 \%$ sequence identity in the $\alpha 1$ domain (Cheng et al. 2012b). PCR amplification of the MHC class I peptide-binding region of Saha-UD alleles identified four Saha-UD alleles (Lane et al. 2012). Saha-UK is expressed in the blood and spleen 


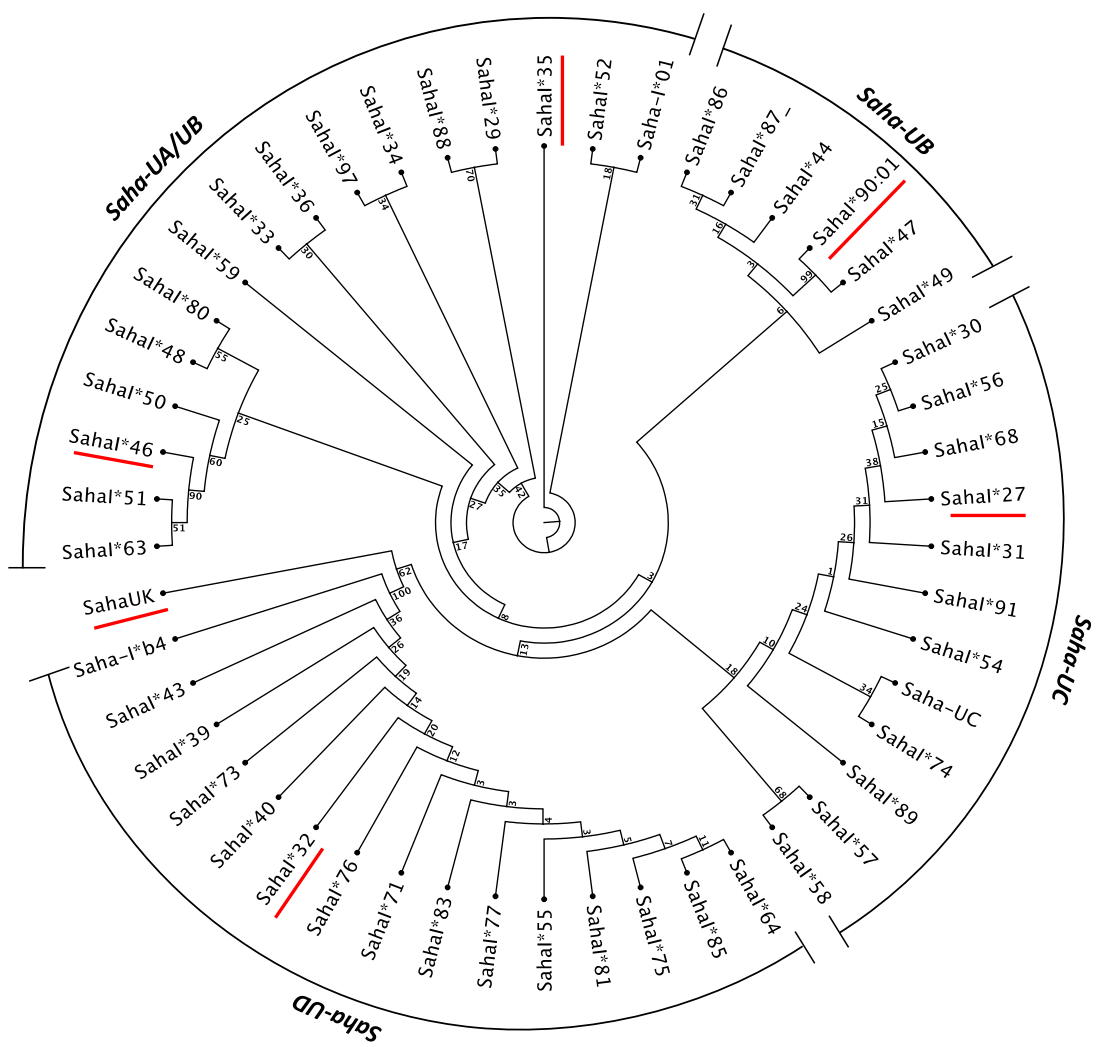

Fig. 2 Phylogenetic analysis of exon 2 of the Tasmanian devil MHC class I alleles deposited to the NCBI database. The Neighbour-Joining method with Jukes Cantor distance measurement (1000 bootstraps) was used for the analysis following the phylogenetic analysis of MHC class I in Cheng et al. (2012a). The following MHC class I sequences covering exon 2 of the class I genes were downloaded from NCBI and trimmed to 180 bp of exon 2, EF591089.1; JN397401.1; JN389437.1; JN389435.1; JN389434.1; GQ411488.1; GQ411484.1; GQ411476.1; GQ411466.1; GQ411464.1; GQ411462.1; GQ411460.1;GQ411458.1; GQ411456.1; GQ411452.1; GQ411448.1; GQ411444.1; GQ411442.1;GQ411438.1; GQ411491.1; GQ411493.1; GQ411489.1; GQ411485.1;GQ411483.1;

(Cheng et al. 2012b) and is orthologous to a suggested nonclassical MHC class I in the closely related marsupials, the grey short-tailed opossum (Monodelphis domestica) and tammar wallaby (Macropus eugenii) (Siddle et al. 2009). Similarly, Saha-UM is orthologous to $-U M$ in the opossum and tammar wallaby. The role of the non-classical MHC class I Saha-UK and Saha-UM is not known, but their conservation between species suggests a marsupial specific function (Siddle et al. 2009; Cheng et al. 2012a). Unlike the other non-classical MHC class I genes, Saha-MR1 has ubiquitous expression (Cheng and Belov 2014) similar to human MR1 (Riegert et al. 1998). Although the role of Saha-MRl in Tasmanian devils has not been studied, it is expected to play the same role as in humans and activate mucosal-associated invariant T (MAIT) cells (KjerNielson et al. 2012; Cheng and Belov 2014). Similarly, it is suggested that $S a h a-C D 1$ presents lipid antigens during microbial infections (Vincent et al. 2003; Cheng and Belov 2014). There are 13 UT family genes located on
GQ411481.1; GQ411479.1; GQ411471.1; GQ411467.1; GQ411465.1;GQ411463.1; GQ411459.1; GQ411457.1; GQ411455.1; GQ411451.1; GQ411447.1;GQ411441.1; GQ411439.1; GQ411437.1; GU363945.1; GQ411454.1; GQ411435.1; JN389438.1; GQ411443.1; GQ411440.1; KY194696.1; GQ411472.1; KT188437.1; JN397398.1; GQ411482.1. MHC class I sequences were excluded if they did not include sequence for exon 2 of the class I gene. The MHC class I gene of each of the clades has been suggested based on the phylogenetic analysis by Cheng et al. (2012a). The MHC class I alleles expressed by DFT1 are underlined in red (colour figure online)

chromosome 1 in the devil (Papenfuss et al. 2015). Three of these UT genes UT2, UT8 and UT11 are expressed in spleen but UTs could not be detected in the lymph node (Papenfuss et al. 2015). Given their restricted expression across different tissue types in Tasmanian devils and other marsupials, as well as limited polymorphism, it is likely that the Saha-UT genes are involved in roles other than antigenic peptide presentation (Krasnec et al. 2016).

\section{Transmission of DFT1 and the role of MHC class I molecules}

Tasmanian devils have reduced genetic diversity at microsatellite loci (Jones et al. 2007), and their MHC class I and class II genes have fewer alleles than observed in other marsupial species, which has been suggested to play a role in allowing tumour cell transmission (Siddle et al. 2007; Cheng et al. 2012a). The classical MHC class I alleles of Saha-UA, and 
Saha-UC share intermediate levels of amino acid identity and Saha-UB has 91-99\% amino acid identity (Lane et al. 2012). Further, 54\% of devils carry a haplotype in which Saha-UA is a pseudogene, leaving these animals with only two polymorphic MHC class I genes (Cheng et al. 2012b). Despite low levels of MHC class I diversity in the population, devils can reject skin grafts in a T-cell mediated response (Kreiss et al. 2011) and the level of MHC class I variation in the population should be sufficient to initiate an immune response against DFT1. However, analysis of ten DFT1 biopsies showed poor infiltration of immune cells, including T-cells, B-cells and dendritic cells, suggesting that the devil immune system is ignorant of the tumour (Howson et al. 2014). Interestingly, MHC class II-positive cells have been observed both within the DFT1 tumour and in the stroma, which may represent macrophages in the tumour tissue.

We have shown that the lack of a T-cell response to DFT1 is due to the loss of MHC class I molecules from DFT1 cells. DFT1 cells contain little MHC class I heavy chain molecules and only trace amounts of $\beta_{2} \mathrm{~m}$ on the cell surface (Siddle et al. 2013). In addition, DFT1 cells do not express MHC class II molecules, but as the cancer derived from a Schwann cell, expression of MHC class II would be unusual. In contrast, Schwann cells in humans and rodents express MHC class I molecules, albeit at low levels, and as such, MHC class I expression would be expected on DFT1 cells (Armati et al. 1990; Meyer Zu Horste et al. 2010). The lack of MHC class I molecules explains the lack of a T-cell response to DFT1 cells, but it does not explain why Natural Killer (NK) cells do not respond to DFT1 due to a missing self ligand.

Loss of MHC class I molecules in DFT1 is due to epigenetic alterations, rather than structural mutations in the DNA (Siddle et al. 2013). The transcripts for $\beta_{2} \mathrm{~m}$, TAP1 and TAP2 are downregulated, but some MHC class I heavy chain is still transcribed (Siddle et al. 2013). While there is no evidence of increased methylation at $\mathrm{CpG}$ sites in the promoter regions of the $\beta_{2} \mathrm{~m}$, TAP1 and TAP2 genes, transcription of these genes can be upregulated when DFT1 cells are treated with the histone deacetylase inhibitor, trichostatin A, which suggests that histone modification, rather than methylation, is involved in MHC class I regulation (Siddle et al. 2013). Further, downregulation of MHC class I expression can be reversed with treatment of DFT1 cells in vitro with interferon gamma (IFN $\gamma$ ), confirming a lack of structural mutations. We have found that MHC class I heavy chain genes, Saha-UA, Saha$U B$ and $S a h a-U C$, are all upregulated in response to IFN $\gamma$ treatment, but notably, Saha-UD does not respond (Caldwell et al. unpublished). This data fits with the finding that there is no IFN $\gamma$ response element in the promoter region of Saha$U D$, in contrast to the other MHC class I heavy chain genes (Cheng et al. 2012b).

Despite the loss of MHC class I molecules from DFT1 cells and high mortality rate among affected devils, there is mounting evidence that the Tasmanian devil immune system is not completely ignorant of DFT1. In addition to the in vitro experiments demonstrating that DFT1 cells upregulate MHC class I in response to IFN $\gamma$, DFT1 cells in tumour biopsies have been found that express $\beta_{2} \mathrm{~m}$ when clusters of CD3positive leukocytes are adjacent, implying a response to IFN $\gamma$ or other inflammatory cytokines (Siddle et al. 2013). Importantly, Pye et al. (2016a) have recently identified four wild Tasmanian devils with DFT1 lesions that regressed over time. These animals had serum antibody responses to $\mathrm{MHC}$ class I-positive DFT1 cells, but not MHC class I negative DFT1 cells. One of these animals also had CD3-positive lymphocytes infiltrating the tumour tissue prior to regression (Pye et al. 2016a). Taken together, these results indicate that the regression of these tumours was immune mediated and potentially MHC class I restricted. Both MHC class I and class II could be involved in this response as it was not determined whether the DFT1 cells were positive for MHC class II. However, it is possible that serum antibodies against $\mathrm{MHC}$ positive DFT1 cells are not always protective as two additional animals were identified with antibody responses and either MHC class I-positive DFT1 cells in the tumour or evidence of lymphocyte infiltration, but these animals did not show tumour regression at the time that they were captured. These animals were not trapped again, so ultimately, their DFT1 status remains unknown.

The ability of DFT1 cells to respond to inflammatory cytokines has led to efforts to utilise MHC class I-positive DFT1 cells as a vaccine and immunotherapy. Immunisation of devils has been performed using a number of strategies, including the use of sonicated DFT1 cells and frozen/thawed, sonicated or irradiated MHC-positive DFT1 cells with an adjuvant of ISCOMATRIX, Poly I:C and CpG (Tovar et al. 2017). While the antibody responses to these strategies varied, responses were only seen in animals immunised with MHC-positive DFT1 cells (Tovar et al. 2017). Interestingly, despite being immunised with MHC-positive cells, the animals raised antibodies against both MHC-negative and MHC-positive DFT1 cells, implying that once the devil immune system is activated, an antibody response is possible against MHC negative cells. This is supported by a recent study showing that devil mononuclear can be cytotoxic to DFT1 cells in vitro (Brown et al. 2016). The sonication of DFT1 cells used in immunisations may be of particular importance for these responses, increasing the number of antigens to which the host immune system is exposed. Despite the presence of serum antibodies, the immunisations with MHC-positive DFT1 cells are not protective against inoculation with DFT1 cells (Tovar et al. 2017).

The Tasmanian devils immunised in the experiments described above were also used for immunotherapy trials once tumours had developed. Significantly, while immunisation was not protective, the tumours appeared more slowly in the immunised animals than in the control animal (Tovar et al. 
2017). Once tumours were palpable, a combination of live or irradiated MHC-positive DFT1 cells and IFN $\gamma$ was injected into the tumours. These protocols resulted in regression of the tumours in three of six animals. These experiments pose unique challenges in an endangered species where access to animals is restricted, and due to the small number of animals in the study, the results are not definitive, but a number of conclusions can be drawn. First, tumour regression after immunotherapy is dependant on immunisation, as a nonimmunised control did not respond to treatment with MHCpositive DFT1 cells once a tumour had formed. Second, the regression of tumours was associated with infiltration of CD3positive cells into the tumour mass and these cells were dominated by CD8-positive T-cells, suggesting an MHC class Irestricted response.

\section{Does MHC genotype affect DFT1 transmission?}

The immune response to DFT1 cells described above suggests that the MHC genotype of host devils may be relevant to DFT1 progression despite the loss of MHC class I from the cell surface. While no correlation has been found between host MHC genotype and susceptibility or resistance to DFT1 (Lane et al. 2012), the evidence that MHC class I expression can be upregulated on DFT1 cells and the presence of serum antibodies to MHC-positive cells suggests that the MHC genotype could play a role, but is perhaps more subtle than simply resistance or susceptibility. The degree of MHC genotype matching between the tumour and host may contribute to alterations in tumour growth rate and degree of immune response. The MHC class I alleles of the animals used in the immunotherapy trials varied and while no specific links between MHC genotype and response could be made, the variation in response to therapy could be due to genetic background (Tovar et al. 2017).

The MHC class I genotype of DFT1 has not been determined conclusively, and the MHC class I alleles reported in DFT1 cells have varied between studies (Table 1). Pye et al. (2016b) have reported that DFT1 has six MHC class I alleles
(SahaI*32, SahaI*35, SahaI*46,SahaI*90, SahaI*45 and SahaI*98), based on sequencing of part of exon 2 of the class I gene from DFT1 biopsies. More recently, it was reported that DFT1 cells have four class I alleles, SahaI*35, SahaI*46, SahaI*90 and SahaI*28 (Tovar et al. 2017). In our own studies of the DFT1 alleles expressed upon stimulation with IFN $\gamma$, we find that SahaI*32, SahaI*35, SahaI*46, SahaI*90 and SahaI*28 are all expressed and represent the minimum MHC class I expressed by DFT1 (Siddle et al. unpublished). Although it is difficult to assign these alleles to specific loci, phylogenetic comparison of the DFT1 alleles to all described devil class I sequences suggests that SahaI*35 and SahaI*46 belong to the Saha-UA gene and SahaI*90 and SahaI*28 belong to the Saha-UB and Saha-UC genes, respectively (Fig. 2; Table 1). SahaI*32 is an established allele for the Saha-UD gene (Siddle et al. 2007). We also find that Saha$U K$ is expressed by DFT1 cells, but as this gene is not polymorphic, it is likely not relevant in this context. In addition, there is no significant expression of the non-classical $\mathrm{MHC}$ class I genes, Saha-UM or CD1 in DFT1 cells (Cheng and Belov 2014).

\section{The role of MHC in other contagious cancers}

The expression of MHC molecules is also of importance in the progression of CTVT. CTVT has a different pattern of progression to DFT1, once CTVT cells are transmitted, there is a growth period followed by a stationary phase and or regression of the tumour (Cohen 1985). The immune response to CTVT has been reviewed in detail elsewhere (Murchison 2008; Siddle and Kaufman 2015), but it is worth noting that CTVT cells are reported to be MHC class I and class II negative during the growth phase, but become positive during regression of the tumours (Hsiao et al. 2004). While there is no definitive study showing that the immune response to CTVT is MHC class I or class II restricted, the correlation of MHC expression and infiltration of lymphocytes into CTVT suggests that this is the case. In addition, a more dated study, involving inoculation of CTVT into dogs with a degree of
Table 1 MHC class I alleles reported in DFT1 and found to be expressed after treatment with $\mathrm{IFN} \gamma$

\begin{tabular}{llll}
\hline MHC class I gene & Pye et al. (2016b) & Tovar et al. (2017) & Upregulated with IFN $\gamma^{*}$ \\
\hline Saha-UA & SahaI*35 & SahaI*35 & Yes \\
Saha-UA/UB & SahaI*46 & SahaI*46 & Yes \\
Saha-UB & SahaI*90 & SahaI*90 & Yes \\
Saha-UC & SahaI*27 & SahaI*28 & Yes \\
& SahaI*98 & & Unknown \\
& SahaI*45 & & Unknown \\
Saha-UD & SahaI*32 & Not reported & No \\
Saha-UK & Not reported & Not reported & Yes \\
\hline
\end{tabular}

Asterisk indicates Siddle unpublished data 
MHC matching, suggested that the genetic background of the host dog, including the MHC genotype, is important for the growth of the tumour (Epstein and Bennett 1974). Thus, the level of MHC mismatch may play a role in the spread of this tumour. Indirect support for this hypothesis comes from the suggestion that CTVT emerged during the domestication of dogs, which could have been associated with a rapid decline in genetic diversity among host dogs (Murchison et al. 2014).

There have been a number of isolated cases of tumour cells successfully passing between individuals in humans. These cases have occurred during transplantation and across the maternal/foetal barrier (Isoda et al. 2009; Yagasaki et al. 2011). As for the established contagious cancers in mammals, these tumour cells have needed to overcome at least partial histocompatibility barriers. In the case of maternal to foetus transfer, loss of the non-inherited maternal HLA has been demonstrated (Isoda et al. 2009), while during transplants, there is usually immunosuppression (van Sandwijk et al. 2013). These examples highlight how quickly regulation of MHC molecules can allow transfer of tumour cells.

As invertebrates, bivalves do not have MHC or antigen receptors and phagocytosis is the primary clearance mechanism used to protect against pathogens. However, terrestrial molluscs are capable of recognising differences in cell surface molecules of self and non-self tissue, rejecting allografts through a macrophage and perforin-induced death (Furuta and Yamaguchi 2011), and it is likely that they have a genetic system for the detection of non-self cells, perhaps similar to the system present in colonial chordates.

\section{Conclusions}

The devil immune system does not respond effectively to DFT1 in the majority of cases, as evidenced by the lack of immune cell infiltration (Howson et al. 2014), high mortality rate (Lachish et al. 2011) and vaccination studies (Kreiss et al. 2015). This is due in part to the lack of MHC class I molecules on DFT1 cells, a feature shared with CTVT and many single organism tumours. An outstanding question related to $\mathrm{MHC}$ class I loss is why NK cells do not target the tumour cells. However, the regulation of MHC class I expression by epigenetic mechanisms is significant as this type of MHC loss can be reversed using epigenetic modifiers and/or inflammatory cytokines, causing MHC molecules to be returned to the cell surface. Regulation of MHC genes may have an evolutionary benefit to a contagious cancer, allowing the host to survive and transmit tumour cells (Siddle et al. 2013). In DFT1, there is now evidence that the devil immune system is capable of responding to these antigens leading to tumour regression in wild species (Pye et al. 2016a) and after vaccination and immunotherapy with MHC-positive DFT1 cells (Tovar et al. 2017).
When MHC class I molecules are upregulated on DFT1 cells, mismatches in MHC alleles between the tumour and host become relevant. As an allograft, DFTs should provide additional antigenic stimuli to host devils when compared to single organism tumours. In humans, the position and number of mismatches at specific HLA correlates with graft failure (Petersdorf 2017). It is likely that the immune response, or lack of, to DFT1 may also be dependant on specific allelic mismatches between the tumour and the host. Due to recent advances in workflows for identifying tumour-specific antigens, peptide vaccines are under intense scrutiny in human cancers (Yadav et al. 2014; Khodadoust et al. 2017) and these have application in DFT1 where polymorphism between the host devils and the tumour could be exploited to develop a targeted peptide vaccine against the disease. By identifying the mismatches in MHC molecules and bound peptides that determine the ability of host animals to respond to the tumour, we may be able to identify the 'line' that allows individuals to distinguish self and non-self.

Acknowledgments The authors thank Annalisa Gastaldello for advice on the manuscript. This work was supported by a Leverhulme Trust project grant (RPG-2015-103) to HVS. AC is supported by a scholarship from the Gerald Kerkut Trust.

Open Access This article is distributed under the terms of the Creative Commons Attribution 4.0 International License (http:// creativecommons.org/licenses/by/4.0/), which permits unrestricted use, distribution, and reproduction in any medium, provided you give appropriate credit to the original author(s) and the source, provide a link to the Creative Commons license, and indicate if changes were made.

\section{References}

Armati PJ, Pollard JD, Gatenby P (1990) Rat and human Schwann cells in vitro can synthesize and express MHC molecules. Muscle Nerve $13: 106-116$

Benacerraf B (1992) The role of MHC gene products in immune regulation and its relevance to alloreactivity. Scand J Immunol 35:376396. doi:10.1111/j.1365-3083.1992.tb02872.x

Benichou G, Takizawa PA, Olson CA et al (1992) Donor major histocompatibility complex (MHC) peptides are presented by recipient MHC molecules during graft rejection. J Exp Med 175:305-308. doi:10.1084/jem.175.1.305

Brown GK, Tovar C, Cooray AA et al (2016) Mitogen-activated Tasmanian devil blood mononuclear cells kill devil facial tumour disease cells. Immunol Cell Biol 94:673-679

Cheng Y, Belov K (2014) Characterisation of non-classical MHC class I genes in the Tasmanian devil (Sarcophilus harrisii). Immunogenetics 66:727-735. doi:10.1007/s00251-014-0804-3

Cheng Y, Sanderson C, Jones M, Belov K (2012a) Low MHC class II diversity in the Tasmanian devil (Sarcophilus harrisii). Immunogenetics 64:525-533

Cheng Y, Stuart A, Morris K et al (2012b) Antigen-presenting genes and genomic copy number variations in the Tasmanian devil MHC. BMC Genomics 13:87. doi:10.1186/1471-2164-13-87

Cohen D (1973) The biological behaviour of the transmissible venereal tumour in immunosuppressed dogs. Eur J Cancer 9:253-258. doi: 10.1016/0014-2964(73)90090-X 
Cohen D (1985) The canine transmissible venereal tumor: a unique result of tumor progression. Adv Cancer Res 43:75-112

Dausset J (1981) The major histocompatibility complex in man. Science 213:1469-1474

Dausset J, Rapaport FT, Machado-Caetano JA (1965) Relationship between grafts and leucocytics or thrombocytics antigens in man. Bibl Haematol 23:104-114

Epstein RB, Bennett BT (1974) Histocompatibility typing and course of canine venereal tumors transplanted into unmodified random dogs. Cancer Res 34:788-793

Furuta E, Yamaguchi K (2011) Transplant rejection in terrestrial molluscs. Invertebr Surviv J 8(1):15-20

Gorer PA (1938) The antigenic basis of tumour transplantation. J Pathol Soc 47:231-252

Gorer PA, Lyman S, Snell GD (1948) Studies on the genetic and antigenic basis of tumour transplantation. Linkage between a histocompatibility gene and "fused" in mice. Proc R Soc Biol 135:499-505

Gould DS, Auchincloss H (1999) Direct and indirect recognition: the role of MHC antigens in graft rejection. Immunol Today 20:77-82. doi: 10.1016/S0167-5699(98)01394-2

Hamede RK, Mccallum H, Jones M (2008) Seasonal, demographic and density-related patterns of contact between Tasmanian devils (Sarcophilus harrisii): implications for transmission of devil facial tumour disease. Austral Ecol 33:614-622. doi:10.1111/j.1442-9993. 2007.01827.x

Hawkins CE, Baars C, Hesterman $\mathrm{H}$ et al (2006) Emerging disease and population decline of an island endemic, the Tasmanian devil Sarcophilus harrisii. Biol Conserv 131:307-324. doi:10.1016/j. biocon.2006.04.010

Howson LJ, Morris KM, Kobayashi T et al (2014) Identification of dendritic cells, B cell and T cell subsets in Tasmanian devil lymphoid tissue; evidence for poor immune cell infiltration into devil facial tumors. Anat Rec 297:925-938. doi:10.1002/ar.22904

Hsiao Y, Liao K, Hung S et al (2004) Tumor-infiltrating lymphocyte secretion of IL- 6 antagonizes tumor-derived TGF- $\beta 1$ and restores the lymphokine-activated killing activity. J Immunol 172:15081514. doi:10.4049/jimmunol.172.3.1508

Isoda T, Ford AM, Tomizawa D et al (2009) Immunologically silent cancer clone transmission from mother to offspring. Proc Natl Acad Sci 106:17882-17885. doi:10.1073/pnas.0904658106

Jones ME, Jarman PJ, Lees CM et al (2007) Conservation management of Tasmanian devils in the context of an emerging, extinctionthreatening disease: devil facial tumor disease. EcoHealth 4:326337. doi:10.1007/s10393-007-0120-6

Karlson RAG, Mann FC (1952) The transmissible venereal tumor of dogs: observations on forty generations of experimental transfers. Ann New Yourk Acad Sci 54:1197-1213

Khodadoust MS, Olsson N, Wagar LE et al (2017) Antigen presentation profiling reveals recognition of lymphoma immunoglobulin neoantigens. Nature 543:723-727. doi:10.1038/nature21433

Kjer-Nielson L, Patel O, Corbett AJ et al (2012) MR1 presents microbial vitamin B metabolites to MAIT cells. Isr Med Assoc J 491:717-725. doi:10.1038/nature11605

Krasnec KV, Papenfuss AT, Miller RD (2016) The UT family of MHC class I loci unique to non-eutherian mammals has limited polymorphism and tissue specific patterns of expression in the opossum. BMC Immunol 17:43. doi:10.1186/s12865-016-0181-9

Kreiss A, Cheng Y, Kimble F et al (2011) Allorecognition in the Tasmanian devil (Sarcophilus harrisii), an endangered marsupial species with limited genetic diversity. PLoS One 6:1-8. doi:10. 1371/journal.pone. 0022402

Kreiss A, Brown GK, Tovar C et al (2015) Evidence for induction of humoral and cytotoxic immune responses against devil facial tumor disease cells in Tasmanian devils ( Sarcophilus harrisii ) immunized with killed cell preparations. Vaccine 33:3016-3025. doi:10.1016/j. vaccine.2015.01.039
Lachish S, Miller KJ, Storfer A et al (2011) Evidence that disease-induced population decline changes genetic structure and alters dispersal patterns in the Tasmanian devil. Heredity (Edinb) 106:172-182. doi:10.1038/hdy.2010.17

Lane A, Cheng Y, Wright B et al (2012) New insights into the role of MHC diversity in devil facial tumour disease. PLoS One 7:1-9. doi: 10.1371/journal.pone.0036955

Liu Z, Sun YK, Xi YP et al (1993) Contribution of direct and indirect recognition pathways to T cell alloreactivity. J Exp Med 177:16431650. doi:10.1084/jem.177.6.1643

Loh R, Bergfeld J, Hayes D et al (2006) The pathology of devil facial tumor disease (DFTD) in Tasmanian devils ( Sarcophilus harrisii ). Vet Pathol 43:890-895

McCallum H, Tompkins DM, Jones M et al (2007) Distribution and impacts of Tasmanian devil facial tumor disease. EcoHealth 4: 318-325. doi:10.1007/s10393-007-0118-0

Metzger MJ, Reinisch C, Sherry J et al (2015) Horizontal transmission of clonal cancer cells causes leukemia in soft-shell clams. Cell 161: 255-263. doi:10.1016/j.cell.2015.02.042

Metzger MJ, Villalba A, Carballal MJ et al (2016) Widespread transmission of independent cancer lineages within multiple bivalve species. Nature 534:705-709. doi:10.1038/nature18599

Meyer Zu Horste G, Heidenreich H, Lehmann HC et al (2010) Expression of antigen processing and presenting molecules by Schwann cells in inflammatory neuropathies. Glia 58:80-92. doi: 10.1002/glia.20903

Murchison EP (2008) Clonally transmissible cancers in dogs and Tasmanian devils. Oncogene 27(Suppl 2):S19-S30. doi:10.1038/ onc. 2009.350

Murchison EP, Tovar C, Hsu A et al (2010) The Tasmanian devil transcriptome reveals Schwann cell origins of a clonally transmissible cancer. Science 327:84-88

Murchison EP, Schulz-Trieglaff OB, Ning Z et al (2012) Genome sequencing and analysis of the Tasmanian devil and its transmissible cancer. Cell 148:780-791. doi:10.1016/j.cell.2011.11.065

Murchison EP, Wedge DC, Alexandrov LB et al (2014) Transmissible dog cancer genome reveals the origin and history of an ancient cell lineage. Science 343:437-440. doi:10.1126/science.1247167

Novinski MA (1876) Zur Frage uber die Impfung der Krebsigen Geschwulste. Zentralbl Med Wissensch 14:790-791

Papenfuss AT, Feng Z-P, Krasnec K et al (2015) Marsupials and monotremes possess a novel family of MHC class I genes that is lost from the eutherian lineage. BMC Genomics 16:535. doi:10.1186/s12864015-1745-4

Pearse AM, Swift K (2006) Allograft theory: transmission of devil facialtumour disease. Nature 439:549. doi:10.1038/439549a

Petersdorf EW (2017) In celebration of Ruggero Ceppellini: HLA in transplantation. Hla 89:71-76. doi:10.1111/tan.12955

Pye R, Hamede R, Siddle HV et al (2016a) Demonstration of immune responses against devil facial tumour disease in wild Tasmanian devils. Biol Lett 12:20160553. doi:10.1098/rsbl.2016.0553

Pye RJ, Pemberton D, Tovar C et al (2016b) A second transmissible cancer in Tasmanian devils. Proc Natl Acad Sci 113:375-379. doi: 10.1073/pnas.1519691113

Riegert P, Wanner V, Bahram S (1998) Genomics, isoforms, expression, and phylogeny of the MHC class I-related MR1 gene. J Immunol 161:4066-4077

van Sandwijk MS, Bemelman FJ, Ten Berge IJM (2013) Immunosuppressive drugs after solid organ transplantation. Neth J Med 71:281-289

Save the Tasmanian Devil Program (2016) DFTD distribution map. http://www.tassiedevil.com.au/tasdevil.nsf/downloads/ A140AACCA1B1F6B0CA2576CB0011BD2C/\$file/DFTD distribution_2016.pdf. Accessed 19 Apr 2017

Siddle HV, Kaufman J (2015) Immunology of naturally transmissible tumours. Immunology 144:11-20. doi:10.1111/imm.12377 
Siddle HV, Kreiss A, Eldridge MDB et al (2007) Transmission of a fatal clonal tumor by biting occurs due to depleted MHC diversity in a threatened carnivorous marsupial. Proc Natl Acad Sci 104:1622116226

Siddle HV, Deakin JE, Coggill P et al (2009) MHC-linked and un-linked class I genes in the wallaby. BMC Genomics 10:310. doi:10.1186/ 1471-2164-10-310

Siddle HV, Kreiss A, Tovar C et al (2013) Reversible epigenetic downregulation of MHC molecules by devil facial tumour disease illustrates immune escape by a contagious cancer. Proc Natl Acad Sci 110:5103-5108. doi:10.1073/pnas.1219920110

Snell GD (1981) Studies in histocompatibility. Science 213:172-178

Snell GD, Kelton D (1953) First chromosome locus in the mouse determining susceptibility and resistance to tumor transplants. Proc Am Assoc Cancer Res 1:53

Strakova A, Murchison EP (2014) The changing global distribution and prevalence of canine transmissible venereal tumour. BMC Vet Res 10:168. doi:10.1186/s12917-014-0168-9
Tovar C, Pye RJ, Kreiss A et al (2017) Regression of devil facial tumour disease following immunotherapy in immunised Tasmanian devils. Sci Rep 7:43827. doi:10.1038/srep43827

Vincent MS, Gumperz JE, Brenner MB (2003) Understanding the function of CD1-restricted T cells. Nat Immunol 4:517-523. doi:10. 1038/ni0603-517

Waanders MM, Heidt S, Koekkoek KM et al (2007) Monitoring of indirect allorecognition: wishful thinking or solid data? Tissue Antigens 71:1-15. doi:10.1111/j.1399-0039.2007.00979.x

Yadav M, Jhunjhunwala S, Phung QT et al (2014) Predicting immunogenic tumour mutations by combining mass spectrometry and exome sequencing. Nature 515:572-576. doi:10.1038/nature14001

Yagasaki H, Ohashi H, Ito M et al (2011) A novel mechanism of transplacental cancer transmission: natural killer/T-cell lymphoma in the paratesticular region is of maternal origin. To the editor. Blood 117: 6046-6048 